\title{
Diseño de sistema de indicadores de gestión para fijar metas en el departamento de facturación
}

\author{
Design of a management indicator system to set goals in the billing department
}
Desenho de um sistema de indicadores de gestão para definir metas no departamento de faturamento

\section{《 Ecaterina Torcates}

torcateecaterina@gmail.com

ORCID: 0000-0001-7676-6174

Universidad de Carabobo, Venezuela

\section{《Usé Ojeda}

joseangel_o@gmail.com

ORCID: 0000-0002-0454-1635

Universidad de Carabobo, Venezuela

\section{《Karelis Moreno \\ kaleism@hotmail.com \\ ORCID: 0000-0002-7273-2003 \\ Universidad de Carabobo, Venezuela}

Recibido: octubre 2019 / Aceptado: noviembre 2019 / Publicado: enero 2020

\section{RESUMEN}

ABSTRACT

El presente estudio se realizó en el Centro Médico, dedicado a ofrecer servicios integrales de salud. El objetivo de la investigación consistió en proponer un diseño de sistema de indicadores de gestión para fijar metas en el departamento de facturación a crédito. Esta investigación se abordó bajo un tipo descriptivo, proyecto factible, enfoque de campo, tipo documental no experimental. La muestra estuvo representada por (23) personas de facturación y cobranza; el estudio fue censal, implementando un instrumento de recolección y la observación directa. Los resultados demostraron que los trabajadores desconocen que los procedimientos utilizados son los más idóneos para el desarrollo de actividades, los clientes reportan altos índices de morosidad. Se concluye que los indicadores de gestión constituyen una herramienta de monitoreo que pueden incorporar mejoras para obtener un excelente desempeño, los aspectos que en conjunto podrían beneficiar el sistema y la puesta en marcha de los indicadores de gestión.

Palabras clave: Facturación a crédito; rotación; sistema, indicadores de gestión
The present study was carried out at the Medical Center, dedicated to offering comprehensive health services. The objective of the research was to propose a design of a management indicator system to set goals in the credit billing department. This research was approached under a descriptive type, feasible project, field approach, non-experimental documentary type. The sample was represented by (23) billing and collection persons; The study was census, implementing a collection instrument and direct observation. The results showed that the workers are unaware that the procedures used are the most suitable for the development of activities; clients report high rates of delinquency. It is concluded that the management indicators constitute a monitoring tool that can incorporate improvements to obtain an excellent performance, the aspects that together could benefit the system and the implementation of the management indicators.

Key words: Billing on credit; rotation; system, management indicators

\section{RESUMO}

\begin{abstract}
O presente estudo foi realizado em Centro Médico, dedicado a oferecer serviços integrais de saúde. O objetivo da pesquisa foi propor o desenho de um sistema de indicadores de gestão para estabelecer metas na área de faturamento de crédito. Esta pesquisa foi abordada sob o tipo descritivo, projeto factível, abordagem de campo, tipo documental não experimental. A amostra foi representada por (23) cobradores e cobradores; O estudo foi censo, implantando instrumento de coleta e observação direta. Os resultados mostram que os trabalhadores desconhecem que os procedimentos utilizados são os mais adequados para o desenvolvimento das atividades, os clientes relatam altos índices de inadimplência. Conclui-se que os indicadores de gestão constituem uma ferramenta de monitoramento que pode incorporar melhorias para a obtenção de um excelente desempenho, aspectos que juntos podem beneficiar o sistema e a implantação dos indicadores de gestão.
\end{abstract}

Palavras chave: Faturamento a crédito; rotação; sistema, indicadores de gestão 


\section{INTRODUCCIÓN}

oda institución comercial con fines de lucro, ha de realizar una gestión eficiente en el departamento que maneja la facturación a crédito de la organización, ya que representa física y fiscalmente el mayor porcentaje de ingresos obtenidos en un período determinado, para que una gestión de cobro y la evaluación en sus resultados sean oportunos, debe existir la planificación, coordinación, ejecución y control de las mismas, siendo el último factor determinante de cada operación, porque permite medir la calidad del trabajo realizado en cuanto al cumplimiento de objetivos y metas.

Tomando en cuenta todos los controles que se pudieran aplicar, el área de ventas y cobros también se ve afectada por factores externos que pueden influenciar en la operatividad de la empresa, como por ejemplo: la crisis económica Venezolana, los desajustes políticos entre otros factores que no pueden ser controlados directamente por la junta directiva de la empresa, sin embargo, es obligación de los mismos monitorearlos constantemente. Por ello es necesario medir la cantidad de días que se mantiene una factura en la empresa antes de ser entregada al cliente, los motivos que lo causaron y posterior a su entrega, los días de vencimiento establecidos entre ambas partes con la finalidad de evitar retrasos en el cumplimiento de obligaciones y metas, asegurando la operatividad de la organización.

Aunado a ello, es preciso afrontar las variables internas y externas que puedan atentar contra el ejercicio económico de la organización. Estas situaciones requieren de la toma de decisiones y según Ayala (2011), indica que las decisiones pueden ser descritas como la respuesta a un problema o la opción entre distintas alternativas para alcanzar unos objetivos Las decisiones que se toman surgen por la necesidad de mitigar o regular dichas variables; en muchas organizaciones se observa como tienen procedimientos de control inadecuados que los llevan frecuentemente al fracaso, las pérdidas y en el peor de los casos a su cierre.

Es por ello que son necesarios utilizar indicadores de gestión con el motivo de alcanzar el éxito de una meta o como medida de corrección de un proceso estudiado; su evaluación es de suma importancia y según, Sánchez (2013), comenta que además de la aplicación de indicadores de gestión cuantitativos financieros, se necesita del uso de indicadores no financieros, criterios lógicos e indicadores cualitativos que aporten los elementos complementarios necesarios para lograr el análisis integral de la gestión, en otras palabras, un indicador de gestión es un instrumento que ubica y pondera el desempeño de objeto de estudio con respecto a un objetivo establecido previamente.

A medida en que las facturas de una empresa avanzan por el departamento con la finalidad de incorporar recaudos necesarios para su posterior envío y cobro, se hace necesario cuantificar los días transcurridos en el departamento con la finalidad de alcanzar un nivel óptimo de días que debe permanecer una factura en el mismo. Este es el caso de del Centro Médico Valle De San 
Diego, C.A., Venezuela, dedicada a ofrecer servicios integrales de salud, la cual creó un proceso llamado "desglose" encargado de armar los expedientes antes de ser enviados a los clientes; el mismo, realiza un proceso posterior a la facturación y trabaja en conjunto con el proceso de cobranza, aumentando así la cantidad de personal involucrado en el proceso.

A pesar de esto, la empresa no evalúa el desempeño de cada uno de los procesos con respecto a metas establecidas por medio de la utilización de indicadores de gestión, solamente se utiliza la comparación de la cifra de facturación y cobranza del mes con respecto a la estimación mensual como meta, haciendo imposible detectar las fallas existentes en la rotación de facturas a crédito del Centro de Salud y la corrección de las mismas.

Al mismo tiempo de la falta de evaluación y control, se encuentran otros factores como la carencia de un sistema adecuado de información que permita una mejor interacción entre los procesos de facturación, desglose y cuentas por cobrar, además de la carencia de pautas o manuales que permitan establecer las actividades que se deben realizar para desarrollar eficientemente los objetivos del Departamento que afecta la facturación a crédito de la Institución.

Es por ello, de vital importancia el diseño de un sistema de indicadores de gestión para la fijación de metas, para medir el grado de eficiencia y eficacia conseguida por el Departamento que afecta la rotación de la facturación a crédito para conseguir oportunidades de mejoras que permitan facilitar el proceso de toma de decisiones en ciertas situaciones con la finalidad de que la empresa logre cumplir sus objetivos.

Para ello, es importante conocer cuál será la herramienta más adecuada y efectiva que permita representar datos fieles a los acontecimientos que se presentan en el Departamento de Facturación a crédito del Centro Médico Valle De San Diego C.A., Venezuela, con la finalidad de fijar metas.

De tal manera, el estudio tiene como objetivo central, proponer un diseño de sistema de indicadores de gestión para la fijación de metas en el Departamento de Facturación a Crédito en el Centro Médico Valle De San Diego, C.A., Venezuela.

\section{Marco teórico}

Catacora (2007), en relación al sistema señala que son un conjunto de elementos, entidades o componentes que se caracterizan por ciertos atributos identificables que tienen relación entre sí, y que funcionan para lograr un objetivo común.

Todo sistema está conformado por un conjunto de elementos que permitan su eficaz funcionamiento, según Andrade (2010), describe cada uno de ellos:

El ambiente es la zona donde interactúan todos los elementos que de una u otra forma, influyen sobre el sistema ya sea, mediante entradas o salidas; las entradas están compuestas por los elementos provenientes del ambiente. Estas entradas representan los insumos solicitados por el sistema para procesarlos y cumplir con sus objetivos; los requerimientos anteriormente descritos requieren de una transformación, lo cual 
aumenta su valor con la finalidad de convertirse en un producto o servicio demandado como insumo por otro sistema. Las salidas hacen referencia de la entrega a terceros de los insumos procesados para cumplir su función y alcanzar los objetivos y la retroalimentación significa el canal mediante el cual se ubican los focos o fallas que se puedan estar generando dentro del sistema, este mediante alertas notifica el elemento afectado. (p. 54).

En tal sentido, es importante resaltar que para el diseño del sistema de indicadores de gestión, es necesario la compresión de la situación actual de la organización donde se lleva a cabo la investigación, la revisión de los objetivos y la identificación de los procesos o elementos que tienen impacto directo en el diseño del nuevo sistema, que determinan la información necesaria para analizar el cumplimiento de los objetivos.

Al respecto Alonso (2008), lo define como un análisis para realizar preguntas y determinar donde se encuentra el error, y así, eliminar primeramente todo lo que no sea necesario; en caso de que no pueda ser eliminado se puede optar por sustituirse. De ser imposible lo anterior, se pasa al siguiente objetivo que es combinar, tomando en consideración que en este paso queda simplificado el trabajo. Por otro lado, se puede mejorar el trabajo si se cambia la secuencia de la actividad o se emplean medios modernos para desempeñar alguna operación.

De esto, es determinante conocer los Indicadores de Gestión, que consiste en comparar el fenómeno de estudio tomando como referencia un patrón preestablecido, al respecto Sánchez (2013), describe un indicador como la relación entre las variables cualitativas y cuantitativas que permite observar una situación y la tendencia de cambio generado en el objeto visualizado, respecto al objetivo y metas previstas e influencias esperadas. Dichos indicadores pueden mostrar resultados en valores, unidades, índices, series estadísticas entre otros.

Un indicador en una medida de la situación ocurrida en un proceso o evento en un momento específico. Su función principal es brindar una visión global de la situación de un determinado proceso, departamento administrativo o incluso de las operaciones de una empresa. Además, pueden ser utilizados para establecer rangos de productividad para el cumplimiento de la misión, objetivos y metas de una operación dada. Como se puede apreciar todas las actividades en cualquier ambiente pueden ser cuantificadas mediante parámetros establecidos, alineados en todo momento con la gerencia estratégica, asegurando así que la organización siga el camino correcto.

En todo caso, los indicadores de gestión brindan información de carácter relevante, pero, estos deben de cumplir con una serie de atributos como son la exactitud, frecuencia, relevancia, integridad, etc. Esta información es requerida ya que actualmente son muchos los factores tanto internos como externos que afectan la operatividad de la empresa y es necesario tomar el control de la situación, conocerla y evitar posibles amenazas y fracasos de la empresa. Por todo lo anteriormente descrito, se puede concluir que los indicadores son necesarios para colaborar con la 
toma de decisiones y se pueden manejar para establecer parámetros y mejorar la operatividad del ambiente donde son utilizados.

Los indicadores de gestión tienen que cumplir con algunos requisitos, elementos para coadyuvar a la operatividad de la empresa y el cumplimiento de sus metas. Estas características según Serna (2008), pueden ser:

La Simplicidad que es la capacidad para definir el evento que se pretende medir, de manera poco costosa en tiempo y recurso, la adecuación entendida como la facilidad de la medida para describir por completo el fenómeno o efecto. Debe reflejar la magnitud del hecho analizado y mostrar la desviación real del nivel deseado; la validez en el tiempo definida como la propiedad de ser permanente en un período deseado, la utilidad comprendida como la posibilidad del indicador para estar siempre orientado a buscar las causas que han llevado a que alcance un valor particular y mejorarlas; y por último la oportunidad entendida como la capacidad para que los datos sean recolectados a tiempo. (p.19)

\section{Elementos de los ilndicadores de gestión}

Sumado a lo expuesto anteriormente, hay elementos importantes que definen a los indicadores de gestión, los cuales necesitan ser incorporados durante su ejecución

- La definición. Expresión que cuantifica el estado de la característica o hecho que se quiere controlar.

- El objetivo. Es la meta que persigue el indicador seleccionado y a la vez señala la mejora que se busca. Entonces, el objetivo facilita la selección de acciones preventivas y correctivas del objeto de estudio.

- Los valores de referencia. La actividad de medir es realizada mediante la comparación y esta no sería posible si no se dispone con un nivel de referencia que proporcione el valor de un indicador.

\section{MÉTODO}

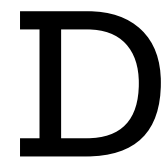

e acuerdo con el problema planteado y en función al objetivo de la investigación, metodológicamente se desarrolló bajo un enfoque cuantitativo, de tipo descriptivo, proyecto factible, diseñado bajo el enfoque trabajo de campo, tipo documental, no experimental. La población estuvo compuesta por veintitrés (23) trabajadores que laboran en el Departamento de Facturación a Crédito de este Centro Médico poseen características comunes tales como: forman parte del equipo de trabajo de la empresa donde se aplicó la investigación, interactúan productos de sus actividades laborales, se encuentran adaptados al sistema de la 
organización, entre otros aspectos que se consideraron fundamentales para recabar la información necesaria y desarrollar el sistema de indicadores de gestión propuesto en esta investigación.

Para recopilar la información se empleó la técnica recolección de datos la encuesta y la observación directa y así, construir el diseño de un sistema de indicadores de gestión, mediante un cuestionario auto administrado porque debe ser llenado por el encuestado, sin intervención del encuestador. Razón por la cual, el formulario o cuestionario fue estructurado por preguntas cerradas con alternativas previamente delimitadas, es decir, si y no; que determinaron la información competente para el diseño del sistema de indicadores de gestión para la fijación de metas propuestos por este estudio.

\section{RESULTADOS}

$\mathrm{L}$ uego de aplicar el instrumento de recolección de datos, permitiendo así, el análisis de detallado de cada una de sus variables con la finalidad de agruparlos y obtener una información confiable. Según Sampieri (2013), la medición de variables primero provee evidencia empírica, el segundo proporciona modelos teóricos para encontrar sentido a ese segmento del mundo real que estamos tratando de describir.

Seguidamente, y en función al objetivo central de la investigación se presentan los resultados obtenidos con la aplicación del instrumento:

Tomando en cuenta el ítem 1, sobre el conocimiento de la misión y la visión del Centro Médico valles de San Diego C.A, Venezuela, se destaca la tabla 1.

Tabla 1. La misión y la visión

\begin{tabular}{rccc}
\hline Alternativas & & Frecuencia & Porcentaje \\
\hline & Sí & 5 & $22 \%$ \\
& No & 18 & $78 \%$ \\
\hline Total & $\mathbf{2 3}$ & $\mathbf{1 0 0 \%}$ \\
\hline
\end{tabular}

Análisis: Como se observa en la tabla 1, el setenta y ocho por ciento (78\%) contestó de manera negativa y el veintidós por ciento (22\%) respondió afirmativamente, lo cual pone en evidencia que la mayor parte de las personas consultadas no está en conocimiento de la misión y visión del Centro Médico.

Adicional, el Ítem 2, sobre los procedimientos de facturación a crédito del Departamento se destaca la tabla 2. 
Tabla 2. Procedimientos de facturación

\begin{tabular}{ccc}
\hline Alternativas & Frecuencia & Porcentaje \\
\hline Sí & 7 & $30 \%$ \\
No & 16 & $70 \%$ \\
\hline Total & $\mathbf{2 3}$ & $\mathbf{1 0 0 \%}$ \\
\hline
\end{tabular}

Análisis: El treinta por ciento (30\%) respondió de manera afirmativa y el otro setenta por ciento $(70 \%)$ negativamente, evidenciándose que un bajo porcentaje de los empleados conocen los procedimientos de Facturación a Crédito dentro del Departamento donde se pone en evidencia la poca coordinación y monitoreo de este proceso dentro de la institución.

Seguidamente, el Ítem 3, sobre las herramientas laborales (reportes) suministradas para el monitoreo de su desempeño en este Departamento, se desarrolla la tabla 3.

Tabla 3 Herramientas laborales (reportes)

\begin{tabular}{ccc}
\hline Alternativas & Frecuencia & Porcentaje \\
\hline Sí & 3 & $13 \%$ \\
No & 20 & $87 \%$ \\
\hline Total & $\mathbf{2 3}$ & $\mathbf{1 0 0 \%}$ \\
\hline
\end{tabular}

Análisis: El trece por ciento (13\%) de las personas consultadas respondió afirmativamente y el ochenta y siente por ciento (87\%) lo hizo de manera negativa, lo cual significa que efectivamente el departamento no se encuentra satisfecho con las herramientas laborales del trabajo. No obstante, las respuestas negativas indican que deben ser mejorados dichos recursos con la finalidad de adecuar la información que allí se presenta.

Sobre el conocimiento de las políticas para el tratamiento de los clientes morosos en este Departamento. Se desarrolló la tabla 4.

Tabla 4. Prevención

\begin{tabular}{ccc}
\hline Alternativas & Frecuencia & Porcentaje \\
\hline Sí & 20 & $87 \%$ \\
No & 3 & $13 \%$ \\
\hline Total & $\mathbf{2 3}$ & $\mathbf{1 0 0 \%}$
\end{tabular}

Análisis: El ochenta y siete por ciento (87\%) contestó afirmativamente y el trece por ciento (13\%) lo hizo de manera negativa, indicando que, según la opinión de la mayor parte de las personas consultadas, efectivamente, los usuarios conocen el tratamiento de clientes que generan 
altos índices de morosidad. De lo antes expuesto, se deduce que existen deficiencias a la hora de otorgar créditos a los clientes, ya que un buen estudio de las características, cualidades, trayectoria y reportes financieros de las personas que acuden a solicitar crédito, por lo menos, reduciría en gran medida los índices de morosidad que actualmente presenta la clínica.

Sobre las líneas de autoridad y responsabilidad dentro del Departamento de Facturación a crédito, se desarrolla la tabla 5.

Tabla 5. Distribución de trabajo y óptima del personal.

\begin{tabular}{ccc}
\hline Alternativas & Frecuencia & Porcentaje \\
\hline Sí & 23 & $100 \%$ \\
No & 0 & $0 \%$ \\
\hline Total & $\mathbf{2 3}$ & $\mathbf{1 0 0 \%}$ \\
\hline
\end{tabular}

Análisis: El cien por ciento (100\%) de las personas consultadas respondió afirmativamente. Lo cual significa que, según la totalidad de la muestra, efectivamente, la información y la comunicación circula de manera fluida por los canales regulares. Por lo tanto, la incorporación del sistema de indicadores de gestión puede obtener ventajas relacionadas al desempeño de cada uno de los procesos del departamento estudiado.

Normas de control interno establecidas para la gestión del Departamento.

Tabla 6. Políticas

\begin{tabular}{ccc}
\hline Alternativas & Frecuencia & Porcentaje \\
\hline Sí & 5 & $22 \%$ \\
No & 18 & $\mathbf{7 8 \%}$ \\
\hline Total & $\mathbf{2 3}$ & $\mathbf{1 0 0 \%}$ \\
\hline
\end{tabular}

Análisis: El setenta y ocho (78\%) respondió de forma negativa indicando que el personal del Departamento Facturación a Crédito de Valle de San Diego C.A., Venezuela, considera que las normas de control interno establecidas para la gestión actual del Departamento deben adecuarse. El restante correspondiente al veintidós por ciento (22\%) de la muestra, considera que dichas normas son adecuadas. Así que, una de las maneras de adecuar y optimizar el control interno es adoptando la propuesta planteada en el presente trabajo de investigación. 
Cumplimiento de los procedimientos administrativos del Departamento de la empresa

Tabla 7. Procesos administrativos

\begin{tabular}{ccc}
\hline Alternativas & Frecuencia & Porcentaje \\
\hline Sí & 0 & $0 \%$ \\
No & 23 & $100 \%$ \\
\hline Total & $\mathbf{2 3}$ & $\mathbf{1 0 0 \%}$ \\
\hline
\end{tabular}

Análisis: Como se observa en el cuadro 7, el cien por ciento (100\%) del personal del Departamento considera que no existen manuales de normas y procedimientos de la empresa. Situación que favorece el diseño del sistema de indicadores de gestión propuesto, porque justamente los trabajadores relacionados con las actividades desarrolladas por el departamento muestran disposición para la realización de las herramientas propuestas.

Cumplimiento de los procesos administrativos (admisión - desglose - cuentas por cobrar) dentro del Departamento de Facturación a Crédito.

Tabla 8. Canales regulares

\begin{tabular}{ccc}
\hline Alternativas & Frecuencia & Porcentaje \\
\hline Sí & 20 & $87 \%$ \\
No & 3 & $13 \%$ \\
\hline Total & $\mathbf{2 3}$ & $\mathbf{1 0 0 \%}$ \\
\hline
\end{tabular}

Análisis: El ochenta y siete por ciento (87\%) respondió de manera afirmativa y el otro trece por ciento (13\%) negativamente, evidenciándose la existencia de una tendencia específica referente a que los procedimientos utilizados son los más idóneos para el desarrollo de las actividades del Departamento de Cuentas por Cobrar de la empresa en cuestión. Por lo tanto, el diseño del sistema de indicadores de gestión puede cubrir la necesidad de mejorar los tiempos para el cumplimiento de los procesos requeridos para el desarrollo eficaz y eficiente de las funciones propias del departamento estudiado. 
Conocimiento de la Organización de políticas para el otorgamiento de créditos.

Tabla 9. Emisión de créditos

\begin{tabular}{ccc}
\hline Alternativas & Frecuencia & Porcentaje \\
\hline Sí & 0 & 0 \\
No & 23 & $100 \%$ \\
\hline Total & $\mathbf{2 3}$ & $\mathbf{1 0 0 \%}$ \\
\hline
\end{tabular}

Análisis: El cien por ciento (100\%) contestó negativamente, lo cual permite afirmar, basado en la opinión de las personas consultadas que, el otorgamiento de créditos a los clientes no favorece la situación financiera de la empresa. Situación que confirma la problemática que dio origen a la investigación y pone en evidencia la necesidad de reestructurar el mencionado proceso con el objeto de acceder de manera óptima a las ventajas que genera un buen manejo de la utilización del crédito, tanto para la organización como para los clientes.

El tiempo prudencial en las instituciones para el proceso de facturación.

Tabla 10. Tiempo de Facturación.

\begin{tabular}{ccc}
\hline Alternativas & Frecuencia & Porcentaje \\
\hline Sí & 6 & $26 \%$ \\
No & 17 & $\mathbf{7 4 \%}$ \\
\hline Total & $\mathbf{2 3}$ & $\mathbf{1 0 0 \%}$ \\
\hline
\end{tabular}

Análisis: Un setenta y cuatro por ciento (74\%) contestó negativamente y el veintiséis por ciento (26\%) de manera afirmativa, lo cual indica que en el Departamento de Cuentas por Cobrar de la empresa estudiada, tiene un proceso de facturación a crédito que no se realiza en un tiempo prudencial. Por ello, se puede afirmar que en Valle de San diego C.A. tiene como deficiencia la ausencia de tiempo prudencial, la cual podría impedir la aproximación de los objetivos del departamento a las metas que sean adoptadas por la empresa. 
El personal involucrado en el proceso de facturación a crédito tiene conocimientos de las políticas para el condicionamiento del cliente

Tabla 11. Condiciones del cliente

\begin{tabular}{ccc}
\hline Alternativas & Frecuencia & Porcentaje \\
\hline Sí & 15 & $65 \%$ \\
No & 8 & $35 \%$ \\
\hline Total & $\mathbf{2 3}$ & $\mathbf{1 0 0 \%}$ \\
\hline
\end{tabular}

Análisis: El sesenta y cinco por ciento (65\%) respondió afirmativamente, mientras el treinta y cinco por ciento (35\%) negativamente, por lo tanto se da a conocer que el personal del departamento involucrado en el proceso de Facturación a crédito tiene conocimientos de estas políticas para el condicionamiento del cliente por lo tanto, se considera que deben participar activamente en el diseño de indicadores de gestión como una mejora para los procesos del departamento.

Aplicación de indicadores de gestiones en el control interno.

Tabla 12. Necesidad de cambio

\begin{tabular}{ccc}
\hline Alternativas & Frecuencia & Porcentaje \\
\hline Sí & 22 & $96 \%$ \\
No & 1 & $4 \%$ \\
\hline Total & $\mathbf{2 3}$ & $\mathbf{1 0 0 \%}$ \\
\hline
\end{tabular}

Análisis: El noventa y seis por ciento (96\%) respondió afirmativamente, indicando que el personal del Departamento de Cuentas por Cobrar Considera que el control interno mediante la aplicación de indicadores de gestión generara un cambio positivo en Valle de San Diego, C.A.

Control para identificar y registrar las transferencias y/o deposito realizado por los clientes?

Tabla 13. Controles

\begin{tabular}{ccc}
\hline Alternativas & Frecuencia & Porcentaje \\
\hline Sí & 23 & $100 \%$ \\
No & 0 & $0 \%$ \\
\hline Total & $\mathbf{2 3}$ & $\mathbf{1 0 0 \%}$ \\
\hline
\end{tabular}


Análisis: El cien por ciento (100\%) del personal encuestado respondió afirmativamente que hay un buen control para identificar y registrar las transferencias y/o deposito realizado por los clientes. Y obtiene una base de datos a partir de la cual podrá contar con la información requerida para la ejecución de un proceso eficaz y eficiente de toma de decisiones.

Parámetros para medir los días de las cuentas por cobrar pendientes

Tabla 14. Implementación de parámetros financieros

\begin{tabular}{ccc}
\hline Alternativas & Frecuencia & Porcentaje \\
\hline Sí & 0 & $0 \%$ \\
No & 23 & $100 \%$ \\
\hline Total & $\mathbf{2 3}$ & $\mathbf{1 0 0 \%}$ \\
\hline
\end{tabular}

Análisis: El cien por ciento (100\%) de las personas consultadas respondió negativamente. Lo cual significa que, según la totalidad de la población, se deben incluir nuevos parámetros para mejorar la relación entre los clientes y el Departamento de Facturación a Crédito.

Indicadores actuales de gestión financieros como herramienta principal para la evaluación de los procesos de facturación a crédito

Tabla 15. Indicadores cualitativos y cuantitativos

\begin{tabular}{ccc}
\hline Alternativas & Frecuencia & Porcentaje \\
\hline Sí & 0 & $0 \%$ \\
No & 23 & $100 \%$ \\
\hline Total & $\mathbf{2 3}$ & $\mathbf{1 0 0 \%}$ \\
\hline
\end{tabular}

Análisis: El cien por ciento (100\%) dijo no, lo cual demuestra que no existe actualmente indicadores de gestión financieros como herramienta principal para la evaluación de los procesos de facturación a crédito, una tendencia precisa sobre el hecho de que se puedan obtener indicadores cuantitativos referidos a las actividades desarrolladas por el departamento estudiado. 
Segregación de funciones que permita el logro de los tiempos de cobro del Departamento de Facturación a Crédito

Tabla 16. Tiempos de cobro

\begin{tabular}{ccc}
\hline Alternativas & Frecuencia & Porcentaje \\
\hline Sí & 3 & $13 \%$ \\
No & 20 & $87 \%$ \\
\hline Total & $\mathbf{2 3}$ & $\mathbf{1 0 0 \%}$
\end{tabular}

Análisis: Como se observa en el ochenta y siete por ciento (87\%) de las personas consultadas respondió negativamente y el trece por ciento (13\%) contestó de forma positiva, evidenciándose que, efectivamente, no existe una adecuada segregación de funciones en el departamento que permita el logro de los tiempos de cobro dentro de la institución para garantizar el buen cumplimiento de sus obligaciones.

Los errores de facturación retrasan las actividades desempeñadas en el Departamento de Facturación a Crédito.

Tabla 17. Actividades Desempeñadas

\begin{tabular}{ccc}
\hline Alternativas & Frecuencia & Porcentaje \\
\hline Sí & 23 & $100 \%$ \\
No & 0 & $0 \%$ \\
\hline Total & $\mathbf{2 3}$ & $\mathbf{1 0 0 \%}$ \\
\hline
\end{tabular}

Análisis: El cien por ciento (100\%) contestó afirmativamente, debido a que las personas del departamento consideran, en su totalidad, que es necesario implementar cambios para minimizar en lo posible los errores en facturación que entorpecen y retardan las actividades de este proceso.

Indicadores de gestión en la toma de decisiones para el mejor desempeño laboral del Departamento de Facturación a Crédito.

Tabla18. Toma de decisiones

\begin{tabular}{ccc}
\hline Alternativas & Frecuencia & Porcentaje \\
\hline Sí & 19 & $83 \%$ \\
No & 4 & $17 \%$ \\
\hline Total & $\mathbf{2 3}$ & $\mathbf{1 0 0 \%}$ \\
\hline
\end{tabular}


Análisis: el ochenta y tres por ciento (83\%) contestó afirmativamente indicando que, la gran mayoría de las personas del departamento consideran que los indicadores de gestión facilitarían la toma de decisiones dentro de este departamento. Resultados que se encuentran a favor de la propuesta del diseño de indicadores de gestión porque una vez cubiertos los diferentes aspectos que exige dicho diseño, el Departamento de Cuentas por Cobrar obtendrá una base de datos a partir de la cual podrá contar con la información requerida para la ejecución de un proceso eficaz y eficiente de toma de decisiones.

Incorporación de mejoras en la información que presentan los reportes de este Departamento de Facturación.

Tabla 19. Monitoreo de actividades

\begin{tabular}{ccc}
\hline Alternativas & Frecuencia & Porcentaje \\
\hline Sí & 20 & $87 \%$ \\
No & 3 & $13 \%$ \\
\hline Total & $\mathbf{2 3}$ & $\mathbf{1 0 0} \%$ \\
\hline
\end{tabular}

Análisis: Como se observar, el ochenta y siete por ciento (87\%) respondió afirmativamente y trece por ciento (13\%) respondió de forma negativa, indicando que más de la mitad del personal del Departamento de Cuentas por Cobrar del Centro Médico Valles de San Diego C.A., Venezuela, está de acuerdo en la incorporación de mejoras en la información presentada en los reportes con el fin de propiciar el monitoreo y seguimiento de las actividades cotidianas. Ya que, el hecho de que los trabajadores del departamento de la organización estudiada, muestren disposición para transformar sus tareas a favor de incrementar mejoras en los procesos de la empresa, resulta un aspecto fundamental para dar fuerza y vigor en actividades desarrolladas.

Frecuencia en el Departamento del análisis de saldo de las cuentas por cobrar.

Tabla 20. Desempeño de procesos

\begin{tabular}{ccc}
\hline Alternativas & Frecuencia & Porcentaje \\
\hline Sí & 2 & $9 \%$ \\
No & 21 & $91 \%$ \\
\hline Total & $\mathbf{2 3}$ & $\mathbf{1 0 0 \%}$ \\
\hline
\end{tabular}

Análisis: de acuerdo a los resultados obtenidos en el ítem 20, indica que un noventa y uno por ciento (91\%) de las personas consultadas respondió negativamente y el nueve por ciento (9\%) lo hizo de manera afirmativa, lo cual significa que efectivamente el Departamento de Facturación a Crédito posee deficiencias en el análisis de saldo de las cuentas por cobrar. 


\section{CONCLUSIONES}

$\mathrm{E}$ n virtud de los resultados al analizar la situación actual en el departamento de facturación a crédito en el Centro Médico, se llegó a la conclusión que se realiza un proceso de facturación crédito no consonó para la situación financiera de la empresa, ya que produce elevados índices de morosidad referente a sus cuentas por cobrar, afectando en forma negativa la liquidez de la organización.

Así mismo, se pudo evidenciar debilidades tales como: el usuario no conoce a fondo el proceso de facturación a crédito, debido a que tienden a pasar por alto facturar algunos ítems o caso contrario duplica la facturación. (ajustes positivos y negativos), no existen políticas referentes a rotación de facturación a crédito, las acciones realizadas para la recuperación de los créditos otorgados a los clientes son deficientes, el período de recuperación de los créditos no genera el flujo de efectivo requerido por la empresa y se tiene desconocimiento de la utilidad de indicadores cuantitativos y cualitativos.

Debido a esto se plantea, De acuerdo a los resultados presentados la investigación generó una propuesta acerca del diseño de un sistema de indicadores para la fijación de las metas del departamento de facturación a crédito en el Centro Médico Valle de San Diego, edo. Carabobo en Venezuela.

Puesto que el proceso de facturación a crédito es un rubro importante para cada organización porque representa la operación principal de una empresa y por ende su principal fuente de ingresos. El establecimiento de un sistema de indicadores de gestión en el departamento que afecten la rotación de la facturación a crédito del centro clínico resulta fundamental para optimizar la gestión y el retorno de la inversión ya que ofrece una solución a la problemática actual en el proceso de Facturación a Crédito y en el departamento, permite una disminución en los días entre la emisión de la factura y su fecha de cobro, un rápido retorno de la inversión, un mayor flujo de efectivo y mayor capacidad para el cumplimiento de sus compromisos, cualidades que en una economía inflacionaria son de vital importancia para la permanencia en el tiempo de la institución

\section{REFERENCIA}

Alonso, J. (2008). Rediseño empresarial: estructura orgánica, procesos y sistemas administrativos y contables. 2da. Edición. Caracas, Venezuela

Andrade, M. (2010). Sistemas y procedimientos. Nuevas tendencias en la Contaduría. 1ra. Edición. Universidad Católica Andrés Bello. Caracas, Venezuela
Ayala, L.(2011), Revista de la Facultad de Ciencias Económicas y Administrativas. Universidad de Nariño. Vol. XII. No. 1, P. 90-112

Catacora, F.(2007). Sistemas y procedimientos contables. Editorial Mac Graw Hill Latinoamericana, S. A. Caracas, Venezuela 
Sampieri, R. (2013). Metodología de la Investigación. 6ta. edición. Mc Graw-Hill/Interamericana editores, S.A. de C.V. México

Sánchez, J (2013). Indicadores de Gestión Empresarial. Palibrio LLC. México.
Serna, H. (2008). Gerencia Estratégica, Índices de Gestión. 3R Editores. Bogotá, Colombia 